\title{
Both sides get the point: Hemispheric sensitivities to sentential constraint
}

\author{
KARA D. FEDERMEIER \\ University of Illinois at Urbana-Champaign, Urbana, Illinois \\ HEINKE MAI \\ University of Magdeburg, Magdeburg, Germany \\ and \\ MARTA KUTAS \\ University of California, San Diego, La Jolla, California
}

\begin{abstract}
Behavioral studies have consistently reported striking differences in the impact of sentence-level information on the processing of words presented in the right (RVF) versus the left (LVF) visual field, with context effects apparent only for RVF items. The consistent lack of such effects in the LVF has been taken to mean that right hemisphere language comprehension is largely insensitive to messagelevel meaning. We used the functional specificity afforded by event-related potential measures to assess this claim. Target words completing strongly and weakly constraining sentence contexts, in which constraint arose at the sentence level rather than from lexical associations, were presented laterally in the LVF or RVF. Increased constraint significantly reduced N400 amplitudes with presentation in both VFs, with no differences in the timing or amplitude of these effects. These results are inconsistent with the view that the VF asymmetries found in behavioral measures reflect differential hemispheric capacities at the level of semantic analysis and integration, although VF-based differences on earlier components (P2) suggest asymmetries in the impact of sentential context on perceptual aspects of word processing in the two hemispheres.
\end{abstract}

Since Paul Broca's discovery (1861) of an association between fluent, articulate speech and the left frontal operculum, research into the neural bases of language has uncovered a complex brain network substrate for the phonological, lexical, semantic, and syntactic functions of normal language comprehension and production (for a review, see Martin, 2003). A particularly salient feature of this brain network is its apparent strong left-laterality: Whereas damage to certain left hemisphere (LH) areas can cause profound and permanent deficits in many language functions, damage to the right hemisphere $(\mathrm{RH})$ homologues of these areas generally leaves basic word and sentence processing relatively intact. This dissociation is one of the most striking and oft-cited examples of hemispheric specialization in humans.

Recently, however, behavioral, neuropsychological, and imaging data have converged to suggest that the $\mathrm{RH}$ may play a larger and more important role in language than has been previously assumed. Data from noninva-

The work reported here was supported by a German National Scholarship Foundation award to H.M. and by National Institute of Child Health and Human Development Grant HD22614 and National Institute on Aging Grant AG08313 to M.K. Correspondence relating to this article can be addressed to K. D. Federmeier, Department of Psychology, 603 E. Daniel, University of Illinois, Champaign, IL 61820 (e-mail: kfederme@ s.psych.uiuc.edu). sive spatial neuroimaging techniques such as positron emission tomography and functional magnetic resonance imaging have revealed language-related activation in brain areas outside the regions classically associated with aphasia, including a number of $\mathrm{RH}$ areas (see, e.g., Ni et al., 2000). In fact, some imaging studies, particularly those focusing on the comprehension of complex narratives (Robertson et al., 2000; St. George, Kutas, Martinez, \& Sereno, 1999) or nonliteral language (Bottini et al., 1994), have found not just bilateral activation patterns, but a predominance of RH activity. One must then ask, not why the RH is unable to process language, but what language functions the RH supports, how they differ from LH functions, and what role they play in normal language processing.

Whereas patients with LH damage often show severe difficulties in the most basic aspects of language, patients with damage to the RH have also been found to show changes in their language comprehension and production. For example, language production after RH damage is marked by socially inappropriate remarks, tangential speech, circumlocutions, and digressions of topic, as well as reduced informational complexity (Joanette, Goulet, \& Hannequin, 1990). Studies of language comprehension in patients with RH damage have noted difficulties extracting the main points from narratives and conversations (Gardner, Brownell, Wapner, \& Michelow, 
1983), appreciating discourse structure (Delis, Wapner, Gardner, \& Moses, 1983; Wapner, Hamby, \& Gardner, 1981), and drawing and revising inferences (Beeman, 1993; Brownell, Potter, Bihrle, \& Gardner, 1986). RH damage has also been associated with problems in processing at least some types of nonliteral language, such as jokes (Brownell, Michel, Powelson, \& Gardner, 1983) and indirect requests (Weylman, Brownell, Roman, \& Gardner, 1989). These findings suggest that although the RH might not be necessary to support the basic functions essential for the processing of individual words or simple sentences, it may make important contributions to higher level meaning processing - for instance by linking words and sentences across larger language units and allowing language information to be used in a flexible and context-sensitive manner.

The RH's contribution to language comprehension in neurologically intact individuals has also been examined by means of the visual half-field (VF) technique (see Banich, 2002, for a review). This technique takes advantage of the fact that stimuli presented in the left half of visual space are initially processed exclusively by (early) visual cortical areas in the $\mathrm{RH}$, and vice versa. In the intact brain, transfer of at least some information across the corpus callosum is possible at later processing stages; however, that information is delayed and likely to be incomplete, because of the relative sparseness of callosal relative to intrahemispheric connections (Berardi \& Fiorentini, 1997) and/or biases and strategies that make it more efficient for the directly stimulated hemisphere to assume more of the processing load (e.g., Banich, 2003). Thus, although the potential contribution of callosal transfer and other factors unrelated to hemispheric dominance to the observed VF patterns must always be given due consideration, the technique is generally believed to skew processing toward the directly stimulated hemisphere. This canonical view seems justified, given that several decades of hemifield studies with brain-intact individuals have documented consistent performance differences for stimuli presented to the right visual field as opposed to the left, and many of these results have been corroborated by evidence from patients with unilateral brain damage and/or from studies of commissurotomized (so-called split-brain) patients.

Research using the VF technique has led to the conclusion that the two hemispheres differ in the meaning that each derives from individual words and sentences. Assessment has largely been based on measures of priming in lexical decision (word/nonword judgment) tasks compared across VFs. For example, with presentation in either VF, response times (RTs) are equally facilitated for target words (e.g., DOG) preceded by a closely related word (cat) or by an unrelated word (table). By contrast, priming for more distantly related word pairs (e.g., goat$\mathrm{DOG}$ ) is greater with LVF/RH presentation (Chiarello, Burgess, Richards, \& Pollock, 1990). Greater facilitation from "summation primes" (three words weakly related to the target) also has been observed for $\mathrm{LVF} / \mathrm{RH}$ presentation (Beeman, Friedman, Grafman, \& Perez, 1994), as well as more priming, at least in some contexts (see Titone, 1998), for the subordinate meanings of ambiguous words (e.g., bridal-vs. freight-TRAIN; Burgess \& Simpson, 1988). These results have been interpreted as suggesting that a critical difference between the hemispheres may lie in the "coarseness" of their semantic coding, with narrow, focused meaning activation in the $\mathrm{LH}$ and weak, diffuse activation in the RH (Beeman, 1998). The broader range of meanings thus "considered" by the RH has been offered as part of the explanation for its role in high-level (and especially nonliteral) language.

However, studies using sentences as primes have led to claims that the RH's language competence is limited to word-level information and lexical associative relationships between words (e.g., Chiarello, Liu, \& Faust, 2001; Faust, 1998). For example, with LVF/RH presentation, lexical decision latencies for sentence-final words were unaffected by scrambling the order of the context words (Faust, Babkoff, \& Kravetz, 1995) or increasing the amount of context from one to three to six words (Faust, Kravetz, \& Babkoff, 1993). In some cases, LVF/RH word processing has even proven insensitive to message-level incongruity (e.g., "The patient parked the MEDICINE"; Faust et al., 1995), although some recent studies have found effects of overt anomalies for LVF processing. For instance, Chiarello et al. (2001) found RT inhibition for anomalous targets in both VFs, as long as the context contained no lexically associated words. Faust, Bar-Lev, and Chiarello (2003) found effects of anomaly for $\mathrm{LVF} / \mathrm{RH}$ targets even in the presence of a lexical associate; however, they also found that in the LVF/RH (unlike the RVF/LH), RT facilitation for congruent words was insensitive to message-level meaning, with equal facilitation for a lexically associated word in a meaningful or in a scrambled or nonsensical context. On the basis of these findings, it has been hypothesized that only the LH can effectively integrate lexical, syntactic, and semantic information to derive the message-level meaning of a multiword utterance. The $\mathrm{RH}$, instead, may be able to note whether or not a word is globally consistent with other words in the context (e.g., toothpaste does not cohere well with the words angry, jury, and confront, as described by Chiarello et al., 2001) but, it is argued, does not construct a higher level meaning representation that is sensitive to factors such as word order or messagelevel constraint.

Clearly, there is an intriguing disparity between the conclusions drawn from neuropsychological studies, which have tended to suggest that intact $\mathrm{RH}$ language functions are particularly crucial for deriving messagelevel meaning, and those drawn from studies of healthy adults, which have concluded that although the RH may be broadly sensitive to word-level meaning, it is generally not able to combine words effectively to construct a message-level representation. One consideration is that the materials and tasks used in the two cases have typically been quite different. In particular, whereas neuropsychological studies have often used untimed measures designed to directly assess comprehension (e.g., true/ 
false questions or choice of story ending), studies with healthy adults have largely used speeded lexical decisions. Given evidence from aphasia suggesting that different processes subserve lexical decision and semantic classification (Bub \& Arguin, 1995), as well as concerns raised from studies of split-brain patients that suggest that lexical decision tasks may underreport the RH's semantic abilities (Baynes \& Eliassen, 1998; Zaidel, 1990), questions remain about the extent to which the observed asymmetries may be task specific.

To circumvent some of these difficulties and to examine language processing asymmetries with a different measure, Federmeier and Kutas (1999a) recorded eventrelated brain potentials (ERPs) in a VF paradigm. ERPs are small voltage fluctuations in the electroencephalogram that are time-locked to sensory, motor, or cognitive events; they are the scalp reflections of synchronous synaptic activity associated with informational transactions in the brain. One advantage of ERPs is that they can be collected while individuals merely read for comprehension, thus avoiding the difficulty of disentangling asymmetries in language processing from asymmetric competencies with specific tasks and the associated decisionmaking and response processes. Another key advantage is that a particular ERP component, the N400, has been closely linked to semantic processing. The N400 is a negative wave peaking around $400 \mathrm{msec}$ after stimulus onset (for a review, see Kutas \& Federmeier, 2001; Kutas \& Hillyard, 1980b) that is elicited in response to all potentially meaningful items, including spoken, written, and signed words, as well as pictures. The amplitude of the N400 shows a strong inverse correlation with the predictability of the eliciting item within its local context (word list, sentence, or discourse; Bentin, McCarthy, \& Wood, 1985; Kutas \& Hillyard, 1984). N400 amplitudes also vary with factors that are related to the ease of accessing information from memory, such as word frequency (Van Petten \& Kutas, 1990), repetition (Nagy \& Rugg, 1989), and semantic memory organization (Federmeier \& Kutas, 1999b). However, the N400 is typically not modulated by grammatical violations and other stimulus and task manipulations that do not affect meaning (Kutas \& Hillyard, 1980a, 1983). It is thus a sensitive and functionally specific measure of meaning access and integration.

In the Federmeier and Kutas (1999a) study, ERPs were recorded as participants read pairs of sentences for comprehension - for example, "He caught the pass and scored another touchdown. There was nothing he enjoyed more than a good game of. ..." One of three types of sentence-final words was presented in the LVF or RVF: "expected exemplars" (e.g., FOOTBALL), "betweencategory violations" (e.g., CHESS), or "within-category violations" (e.g., BASEBALL). Two key findings emerged from this study and a follow-up using sentence-final pictures (Federmeier \& Kutas, 2002): First, N400 responses in the two VFs were equally affected by congruencythat is, reduced for expected exemplars relative to between-category violations. Indeed, the responses to these two ending types were statistically indistinguishable in the two VFs. Thus, there was no evidence to suggest that LVF processing was less affected by messagelevel plausibility. However, a striking VF-based difference was observed in the N400 response to within-category violations, or unexpected words from the same semantic category as the expected endings. When presented to the $\mathrm{RVF} / \mathrm{LH}$, these violations elicited N400 responses of intermediate amplitude. However, in the LVF/RH, responses to these items were no different from responses to the between-category violations. Since the contextual (im)plausibility of the within- and between-category violations was matched on average, $\mathrm{LVF} / \mathrm{RH}$ responses in this case were seemingly even more affected than RVF/ LH responses by a word's fit to the message-level meaning of the sentence.

The N400 data, therefore, cohere with neuropsychological data to suggest that message-level information impacts processing in both VFs, and by inference, in both hemispheres. The root of language processing asymmetries would thus seem to lie not in whether each hemisphere constructs a message-level representation of the context, but how each uses message-level information to guide the processing of subsequent words. Federmeier and Kutas (1999a), however, compared responses to plausible and implausible sentence pair completions. Although care was taken to exclude lexical associates from the targetcontaining sentences, it remains possible that word-level information alone might have provided sufficient cues for expected and unexpected completions to be distinguished in the LVF/RH. As a much stronger test of the claim that message-level information does not impact processing for LVF/RH stimuli, therefore, the present study uses ERPs to examine the effects of sentential constraint on the processing of words presented to each VF.

Participants were asked to read for comprehension a set of entirely congruent sentences that differed only in the extent to which the sentence-final (target) word was constrained by message-level meaning information - for instance:

Strongly constrained: "She was suddenly called back to New York and had to take a cab to the AIRPORT."

Weakly constrained: "She was glad she had brought a book since there was nothing to read at the AIRPORT."

Numerous behavioral and ERP studies have shown that increased constraint is associated with increased facilitation for word processing, in the form of faster RTs in lexical decision tasks (e.g., Schwanenflugel \& Shoben, 1985 ) and reduced N400 responses (Kutas, Lindamood, \& Hillyard, 1984). These effects of message-level constraint on the N400 have been shown to occur independently of lexical associative effects (Van Petten, 1993). In the present study, the same words were used as (plausible) completions of strongly and weakly constraining sentences, and lexical-associative relationships within those sentences were minimized. The conditions thus differ only in how strongly the target words are predicted 
by the sentence message-level information. With presentation to the RVF/LH, we expect to replicate the N400 constraint effects observed for these types of materials with central presentation. However, if the VF-based differences observed in behavioral studies arise because $\mathrm{LVF} / \mathrm{RH}$-initiated semantic processing is insensitive to message-level meaning information (Chiarello et al., 2001; Faust et al., 2003), N400 constraint effects should not be observed (or should be strikingly reduced) with initial presentation to the $\mathrm{LVF} / \mathrm{RH}$.

In addition to looking at constraint and VF effects on semantic processing, as indexed by the amplitude and latency of the N400 response, we examined earlier components of the ERP linked to perceptual analysis. When pictures were used as sentence endings (Federmeier \& Kutas, 2002) and were presented initially to the RVF/LH, we found congruency effects on the P2 component, a frontally distributed positive-going potential that peaks around $200 \mathrm{msec}$. P2 amplitude modulations have been linked to the detection and analysis of visual features in selective attention tasks (Hillyard \& Münte, 1984; Luck \& Hillyard, 1994), with increased amplitudes for stimuli containing the target feature. For stimuli in the RVF (but not in the LVF), P2 amplitudes were larger for expected than for unexpected endings, suggesting that context can provide top-down information allowing for more efficient visual feature extraction from expected targets, but only for stimuli initially projected to the LH. Here we examined whether similar top-down effects can be observed for visual word processing, as a function of contextual constraint.

\section{METHOD}

\section{Materials}

Stimulus materials consisted of 160 sentence-final target words embedded in 160 weakly and 160 strongly constraining sentence contexts. Eight strongly constraining sentence contexts and 49 weakly constraining sentence contexts were taken from published sources that reported cloze probabilities for sentences (Bloom \& Fischler, 1980; Griffin \& Bock, 1998; Rayner \& Well, 1996; Sanocki \& Oden, 1984). ${ }^{1}$ The remaining 152 strongly constraining and 111 weakly constraining sentence contexts were chosen from an unpublished set. Cloze probabilities for these sentences were obtained from 40-80 UCSD undergraduate students (across different sessions). The student volunteers were asked to complete each sentence context with "the first word that comes to mind." Weakly constraining sentences (from all sources) were defined as sentences with no more than $40 \%$ agreement (range: $16 \%-40 \%$ ) for the most common completion, whereas strongly constraining sentences (from all sources) were defined as those with at least $70 \%$ agreement (range: $70 \%-100 \%$ ) for the most common completion. Mean sentence length (in number of words) was matched for strongly and weakly constraining contexts. In addition, strongly constraining sentences were chosen such that the constraint on the sentence-final target word arose from world knowledge at the sentence message level and not as a function of simple lexical association. Only 12 of the 160 sentences $(7.5 \%)$ contained any lexical item with an associative strength (Kiss, Armstrong, Milroy, \& Piper, 1973) of greater than .20 with the sentence-final target word. These associated items were always at least two words away from the target, with a mean distance of seven words. Since lexical priming effects have been shown to dissipate quickly (Gough, Alford, \& Holley-Wilcox, 1981; Ratcliff \& McKoon, 1988), any observed facilitation for the target words in strongly constraining sentences is very unlikely to be driven by word-level priming alone.

Strongly constraining sentence contexts were paired with the ending obtaining the highest cloze probability for that context (always $70 \%$ or above). These same 160 words were then paired with the weakly constraining sentence contexts to yield plausible, lowcloze-probability endings (cloze probability always $40 \%$ or below). Across the experiment, therefore, sentence-final target words in the two constraint conditions were perfectly matched for all lexical variables (mean length $=5$ letters, $S E=0.1$; mean frequency [Francis $\&$ Kučera, 1982] $=118, S E=15$ ). The strongly and weakly constraining sentences were then divided into halves and combined in two lists of 160 sentences each, such that the same target word was not repeated in a list. Half of each context type within each list was shown to the LVF and half to the RVF, counterbalanced across participants. Within each list, sentence-final targets were matched for word length and frequency (Francis \& Kučera, 1982) across both constraint and VF conditions. The Appendix gives examples of the stimuli.

\section{Participants}

Thirty-two UCSD undergraduate volunteers (16 men and 16 women, 18-24 years of age, mean age $=19$ ) participated in the experiment for cash or class credit. All were right-handed, as assessed by the Edinburgh Inventory (Oldfield, 1971); 4 reported having a left-handed member of their immediate family. All were monolingual English speakers with no history of reading difficulties or neurological/psychiatric disorders. Eight participants were randomly assigned to each of the four stimulus lists.

\section{Procedure}

The volunteers were tested in a single experimental session conducted in a soundproof, electrically shielded chamber. They were seated in a comfortable chair $40 \mathrm{in.} \mathrm{in} \mathrm{front} \mathrm{of} \mathrm{a} \mathrm{monitor} \mathrm{and} \mathrm{were}$ instructed to read the stimulus sentences for comprehension.

The presentation of each sentence was preceded by a series of crosses (500-msec duration, with a stimulus onset asynchrony varying randomly between 1,000 and $1,500 \mathrm{msec}$ ) to orient the volunteer toward the center of the screen. Sentences were then presented one word at a time horizontally for a duration of $200 \mathrm{msec}$, with a stimulus onset asynchrony of $500 \mathrm{msec}$. Non-sentence-final words were presented in the center of the screen, but sentence-final words were presented pseudorandomly ${ }^{2}$ in the left or right visual hemifield with the inner edge $2^{\circ}$ of visual angle from fixation. A central fixation point remained visible throughout the trial, positioned a half degree below the bottom-most edge of the centrally presented words. The volunteers were asked not to blink or move their eyes during sentence presentation. The final, target word was followed by a blank screen for 3,000 msec, after which the next sentence appeared automatically.

The volunteers were given a short break after every 20 sentences. At the conclusion of the recording session, the participants were given a surprise recognition memory test over the sentence-final target words. The 160 target words were mixed with 160 new words not previously seen in any part of the experimental session. New words were matched for length, frequency, and part of speech with the target words. The participants were asked to circle all of the words they remembered seeing in the experiment.

\section{EEG Recording Parameters}

The electroencephalogram (EEG) was recorded from 26 tin electrodes embedded in an Electro-Cap (Electro-Cap International, Eaton, $\mathrm{OH}$ ) referenced to the left mastoid. These sites included midline prefrontal (MiPf), left and right medial prefrontal (LMPf and RMPf), left and right lateral prefrontal (LLPf and RLPf), left and 
right medial frontal (LMFr and RMFr), left and right mediolateral frontal (LDFr and RDFr), left and right lateral frontal (LLFr and RLFr), midline central (MiCe), left and right medial central (LMCe and RMCe), left and right mediolateral central (LDCe and RDCe), midline parietal $(\mathrm{MiPa})$, left and right mediolateral parietal (LDPa and RDPa), left and right lateral temporal (LLTe and RLTe), midline occipital (MiOc), left and right medial occipital (LMOc and RMOc), and left and right lateral occipital (LLOc and RLOc). Blinks and eye movements were monitored via electrodes placed on the outer canthus (left electrode serving as reference) and infraorbital ridge of each eye (referenced to the left mastoid). Electrode impedances were kept below $5 \mathrm{k} \Omega$. The EEG was processed through Grass amplifiers (Grass-Telefactor, West Warwick, RI) set at a bandpass of $0.01-100 \mathrm{~Hz}$, was continuously digitized at $250 \mathrm{~Hz}$, and was stored on hard disk for later analysis.

\section{Data Analysis}

Data were rereferenced offline to the algebraic average of the left and right mastoids. Trials contaminated by eye movements, blinks, excessive muscle activity, or amplifier blocking were rejected offline before averaging; approximately $15 \%$ of trials in each hemifield were lost because of such artifacts. ERPs were computed for epochs extending from $100 \mathrm{msec}$ before to $920 \mathrm{msec}$ after stimulus onset. Averages of artifact-free ERP trials were calculated for target words in the two constraint conditions (strongly or weakly constraining) in each hemifield (RVF or LVF) after subtraction of the 100-msec prestimulus baseline. ERPs were filtered with a digital bandpass filter of $0.2-20 \mathrm{~Hz}$ prior to measurement and analysis. All $p$ values are reported after epsilon correction (Huynh-Feldt) for repeated measures with more than one degree of freedom.

\section{RESULTS}

\section{Behavior}

Volunteers correctly identified as "old" an average of $46.8 \%$ of the sentence-final target words on the recognition memory test, with a false alarm rate of $0.09 \%$ (note that there is only a single false alarm rate, since test items were not lateralized). $d^{\prime}$ was 1.45 across both VF conditions (1.48 for stimuli presented in the RVF and
1.42 for those in the LVF). Participants were thus able to differentiate words studied in both VFs from new words, indicating that they must have generally been able to read the peripherally presented words in both the left and right visual fields.

To examine the influences of VF and constraint on recognition memory, hit rates were subjected to an omnibus 2 (visual field: RVF vs. LVF) $\times 2$ (sentential constraint: strongly vs. weakly constraining) ANOVA. There was a main effect of sentential constraint $[F(1,31)=4.71$, $p<.05]$, with better recognition memory for targets that had been viewed in weakly rather than in strongly constraining contexts, and a marginal effect of visual field $[F(1,31)=3.26, p=.08]$, with somewhat better recognition for targets presented in the RVF. As can be seen in Figure 1, these effects were modulated by a significant interaction between constraint and visual field $[F(1,31)=$ $6.66, p=.01]$. Planned comparisons revealed an effect of constraint on recognition memory for target words when presented to the RVF $(t=3.67, p<.001)$, with better recognition memory for words that had been seen in weakly rather than in strongly constraining sentences, but no effect of constraint for words presented to the LVF $(t=0.39, p=.70)$. There was no significant difference in hit rates as a function of visual field for targets that had been embedded in strongly constraining contexts $(t=0.98, p=.33)$, but in weakly constraining contexts, recognition memory was better for words presented in the RVF $(t=3.02, p<.01)$. Overall, then, recognition was selectively improved for words that had been viewed in weakly constraining contexts and were presented initially to the LH (RVF).

\section{Event-Related Potentials}

Grand average ERPs to sentence-final target words in each VF are shown in Figure 2. Early components in all

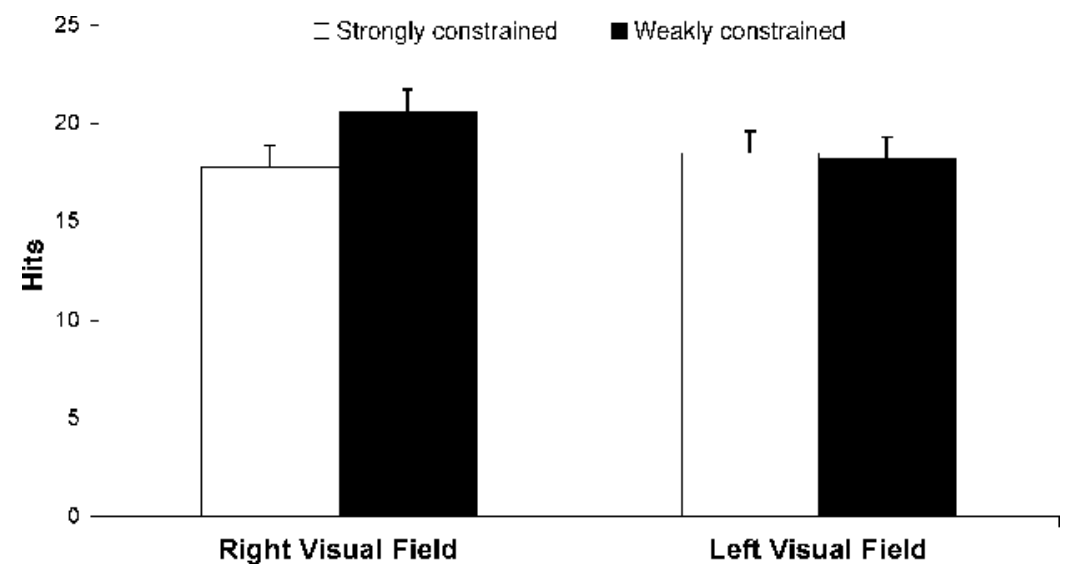

Figure 1. Recognition accuracy. Mean hit rates (with standard errors) are plotted for recognition of target words as a function of $\mathrm{VF}$ in which they were originally viewed and constraint provided by the context in which they originally appeared. Recognition rates were highest for target words presented in weakly constraining contexts to the RVF/LH. 


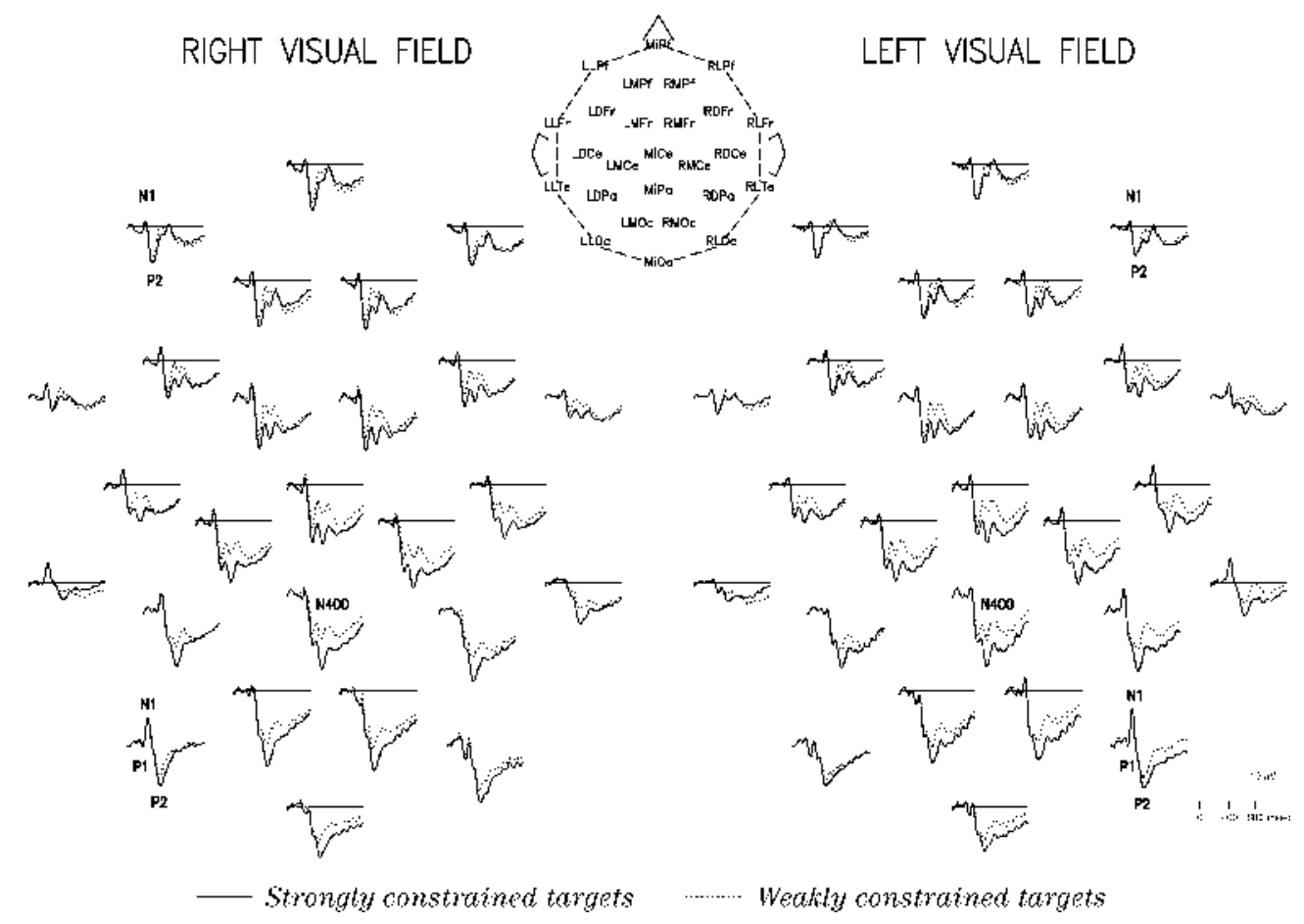

Figure 2. ERP constraint effects. Grand average ERP waveforms are shown for target words in strongly and weakly constraining contexts at all 26 electrode sites. The head diagram shows the electrode arrangement. ERPs elicited during left hemisphere (RVF) presentation are shown on the left and those elicited during right hemisphere (LVF) presentation are shown on the right. Negative is plotted up here and in all subsequent plots. Responses to visual words in both VFs are characterized by the same set of early components $(\mathrm{P} 1, \mathrm{N1}, \mathrm{P2})$, and N1 amplitudes are lateralized as expected for lateralized stimulus presentation. For presentation in both VFs, responses in the 300- to 500-msec time window (N400) were more negative for target words in weakly rather than in strongly constraining contexts. In addition, with RVF presentation, responses over frontal sites in the 200- to 300-msec time window (P2) were more positive for strongly than for weakly constrained targets.

conditions and hemifields include, at posterior sites, a positivity (P1) peaking around $80 \mathrm{msec}$, a negativity (N1) peaking between 150 and $200 \mathrm{msec}$ (depending on VF and channel), and a positivity (P2) peaking around $225 \mathrm{msec}$. As expected, these effects are strongly lateralized, being most prominent over posterior sites contralateral to the visual half-field of presentation. At frontal sites, all conditions include a negativity (N1) peaking around $150 \mathrm{msec}$ and a positivity (P2) peaking around $250 \mathrm{msec}$. Early components are followed, most prominently for targets in weakly constraining sentence contexts, by a broadly distributed negativity (N400) between 300 and $500 \mathrm{msec}$ and, in both conditions, by a posterior, lateralized negativegoing effect extending from about $300 \mathrm{msec}$ to the end of the epoch.

ERPs were measured and analyzed in four time windows. To confirm the success of the lateralized presentation, visual N1 responses (100-200 msec) were analyzed for lateralization over the back of the head, where this component tends to be largest (channels LDCe, RDCe, LDPa, RDPa, LMOc, RMOc, LLTe, RLTe, LLOc, and RLOc). The sustained posterior negativity was also measured at these same channels between 300 and $900 \mathrm{msec}$.
Then, in order to examine the influence of the experimental variables on perceptual and semantic processing, frontal P2 responses (200-300 msec) were measured over anterior channels (MiPf, LLPf, RLPf, LMPf, RMPf, LDFr, RDFr, LLFr, RLFr, LMFr, and RMFr) and N400 responses (300-500 msec) were measured over all channels, and both were analyzed as functions of $\mathrm{VF}$ and constraint.

Visual N1 and selection negativity. Figure 3 shows ERP responses at posterior channels to words presented in the right and left visual fields, collapsed across constraint. To confirm that lateralized presentation of the stimuli resulted in the expected lateralization of visual processing, mean amplitude N1 responses (100-200 msec) were measured at 10 posterior channels. These measures were subjected to an omnibus 2 (visual field: RVF vs. LVF) $\times 2$ (hemisphere: left vs. right) $\times 5$ (electrode) ANOVA. There were no main effects of either visual field $[F(1,31)=1.24, p=.27]$ or hemisphere $[F(1,31)=$ $1.05, p=.31]$, but there was a significant interaction of the two $[F(1,31)=87.74, p<.001]$. As expected, for RVF presentations, N1s were larger over left $(-1.19 \mu \mathrm{V})$ than over right $(0.58 \mu \mathrm{V})$ scalp sites, and for LVF presentations, N1s were larger over right $(-1.69 \mu \mathrm{V})$ than 
LEFT OCCIPITAL SITE

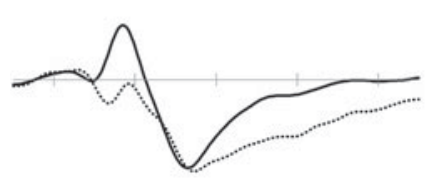

Right visual field
Left visual field
RIGHT OCCIPITAL SITE
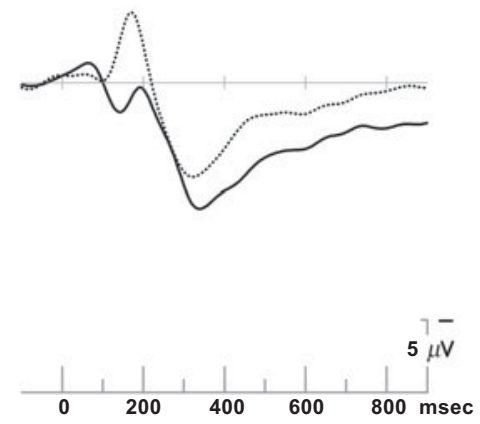

Figure 3. Effects of stimulus lateralization on ERP. To show the general ERP effect of stimulus lateralization, responses to target words (collapsed across constraint) presented to the RVF and LVF are overplotted at left and right occipital scalp sites (where such effects were largest). N1 responses (100-200 msec) are largest contralateral to $\mathrm{VF}$ of presentation and are followed from about $300 \mathrm{msec}$ by an extended negative-going effect that is also largest contralaterally.

over left $(0.63 \mu \mathrm{V})$ scalp sites. N1 responses were also delayed over ipsilateral relative to contralateral sites by an average of $10 \mathrm{msec}[F(1,31)=38.8, p<.001]$, reflecting callosal transfer.

Previous studies measuring ERPs to lateralized visual stimuli have reported a sustained late negative-going effect over lateral and posterior scalp sites contralateral to the VF of stimulus presentation (Federmeier \& Kutas, 1999a, 2002; Neville, Kutas, \& Schmidt, 1982), and we also observed a visually similar effect in this study. To characterize this negative-going process, mean amplitude measures from 300-900 msec were taken from the same 10 posterior electrodes and were subjected to an omnibus 2 (visual field: RVF vs. LVF) $\times 2$ (hemisphere: left vs. right scalp sites) $\times 5$ (electrode) ANOVA. There was a main effect of hemisphere $[F(1,31)=10.29, p<$ $.01]$, with greater negativity over left than over right scalp sites, as well as a nonsignificant tendency for more negative responses with LVF presentation $[F(1,31)=$ $2.11, p=.16]$. As expected, visual field and hemisphere interacted $[F(1,31)=100.11, p<.001]$, with greater negativity over scalp sites contralateral to the VF of presentation.

In summary, we observed the expected general effects of stimulus lateralization on both early and later ERP responses.

Frontal P2. As was described in the introduction, frontal P2 responses have been associated with higher order perceptual and attention-related processing. To examine the effects of VF and constraint on these processes, mean amplitude measures were taken from 200 $300 \mathrm{msec}$ at the 11 anterior scalp sites. Figure 4 shows these responses (at two representative frontal channels) for each VF as a function of constraint. These measures were subjected to an omnibus 2 (visual field: RVF vs. LVF) $\times 2$ (sentential constraint: strongly vs. weakly constraining) $\times 11$ (electrode) ANOVA. There were main effects of both visual field $[F(1,31)=5.33, p=.03]$ and sentential constraint $[F(1,31)=19.31, p<.001]$, with larger overall $\mathrm{P} 2$ responses to items presented in the RVF and for targets in strongly constraining contexts. These main effects were modulated by a visual field $\times$ constraint interaction $[F(1,31)=4.02, p=.05]$. Planned comparisons revealed that $\mathrm{P} 2$ amplitudes did not differ as a function of visual field for targets in weakly constraining sentences $[F(1,31)=0.25, p=.62]$ but were larger with RVF $(5.67 \mu \mathrm{V})$ than with $\operatorname{LVF}(4.51 \mu \mathrm{V})$ presentation for targets in strongly constraining sentences $[F(1,31)=8.32, p<.01]$. Overall, constraint strongly modulated P2 amplitudes for initial presentation to the LH (RVF): 5.67 versus $3.97 \mu \mathrm{V}$ in strongly versus weakly constraining contexts, respectively $[F(1,31)=21.07, p<$ $.001]$. The effect of constraint on P2 amplitudes with LVF presentation was smaller -4.51 versus $3.79 \mu \mathrm{V}-$ and was only marginally significant $[F(1,31)=3.86$, $p=.058]$.

In summary, the presence of a strongly constraining sentence context affected word processing between 200 and $300 \mathrm{msec}$ post-stimulus onset, presumably at a perceptual level, more strongly for targets presented initially to the LH (RVF) rather than to the RH (LVF).

N400. To examine the effects of VF and constraint on the semantic processing of target words, mean amplitude measures were taken from $300-500 \mathrm{msec}$ at all 26 electrode sites. These measures were subjected to an omnibus 2 (visual field: RVF vs. LVF) $\times 2$ (sentential constraint: strongly vs. weakly constraining) $\times 26$ (electrode) ANOVA. There was a main effect of visual field $[F(1,31)=5.84$, $p=.02]$, with more negative responses to words presented in the LVF. ${ }^{3}$ There was also a main effect of sentential constraint $[F(1,31)=67.06, p<.001]$, with greater $\mathrm{N} 400$ amplitudes to targets in weakly $(2.94 \mu \mathrm{V})$ rather than strongly $(5.40 \mu \mathrm{V})$ constraining contexts. There was no interaction between visual field and sentential constraint $[F(1,31)=0.01, p=.91]$ and no hint of such an interaction even over a more restricted subset 

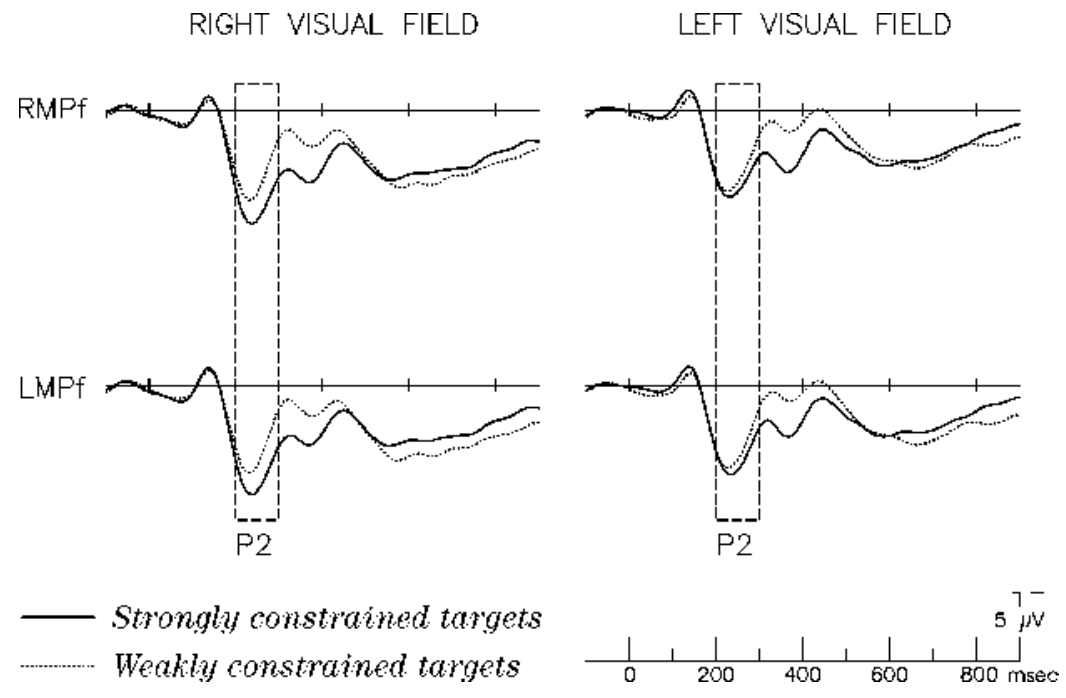

Figure 4. Effects of constraint on the P2 component. Responses are shown to target words in strongly and weakly constraining contexts at right (RMPf) and left (LMPf) medial prefrontal sites (where $P 2$ effects tend to be largest). Responses with left hemisphere (RVF) presentation are on the left and those with right hemisphere (LVF) presentation are shown on the right. For RVF presentation, P2 responses (200-300 msec) were significantly larger (more positive) for target words in strongly rather than weakly constraining contexts.

of electrodes [visual field $\times$ sentential constraint $\times$ electrode: $F(25,775)=0.54, p=.97]$.

Planned comparisons were conducted within each visual field, using an omnibus 2 (sentential constraint: strongly vs. weakly constraining) $\times 26$ (electrode) ANOVA. These analyses revealed a significant effect of sentential constraint in both visual fields. For RVF presentation, mean amplitudes were $5.73 \mu \mathrm{V}$ to targets in strongly constraining sentences and $3.30 \mu \mathrm{V}$ to targets in weakly constraining sentences $[F(1,31)=40.00, p<.001]$. For LVF presentation, mean amplitudes in the same comparison were 5.07 and $2.58 \mu \mathrm{V}$, respectively $[F(1,31)=$ $35.50, p<.001]$. In order to compare responses at each constraint level as a function of VF, planned comparisons were conducted using the four central channels (LMCe, RMCe, MiCe, and MiPa); these channels were chosen because N400 effects are typically maximal at these channels and because using a more restricted set of electrodes helps minimize overlap with the lateralized, posterior effect already described. For targets in strongly constraining sentence contexts, an omnibus 2 (visual field: RVF vs. LVF) $\times 4$ (electrode) ANOVA revealed no main effect of visual field $[F(1,31)=0.36, p=.55]$. The same analysis on the targets in the weakly constraining sentence contexts also revealed no effect of visual field $[F(1,31)=1.05, p=.31]$.

To further characterize the distribution and latency of the N400 constraint effect, difference ERPs were created for each VF condition by doing a point-by-point subtraction of targets in strongly constraining sentences from targets in weakly constraining sentences (Figure 5). This procedure removes effects of stimulus lateralization that are consistent across experimental conditions (e.g., N1 effects and the sustained posterior difference), allowing a comparison of the N400 constraint effect across VFs. Mean amplitude of the constraint effect was measured between 300 and $500 \mathrm{msec}$ at 16 scalp electrodes ${ }^{4}$ and subjected to an omnibus 2 (visual field: RVF vs. LVF) $\times$ 2 (hemisphere: left vs. right scalp sites) $\times 2$ (laterality: lateral vs. medial scalp sites) $\times 4$ (anteriority: prefrontal, frontal, parietal, or occipital) ANOVA. There was no effect of visual field $[F(1,31)=0.01, p=.94]$ and no interactions of visual field with hemisphere $[F(1,31)=1.24$, $p=.27]$, laterality $[F(1,31)=0.04, p=.84]$, or anteriority $[F(3,93)=0.54, p=.58]$. Effects in both VFs had the general distributional pattern characteristic of the N400: biggest over medial and posterior sites and with a rightlateralized skew. There was, however, a significant visual field $\times$ hemisphere $\times$ anteriority interaction $[F(3,93)=$ $4.68, p=.01]$. For LVF presentation, the rightward skew of the scalp distribution of the N400 increased steadily as one moved from more frontal to more posterior sites, reaching its maximum over the back of the head. For RVF presentation, the rightward skew was similar over frontal sites but was at its minimum over the most posterior sites. Thus, as can be seen in Figure 6, at the most posterior sites N400 effect distributions were shifted contralateral to the VF of presentation, with greater right-lateralization of the N400 for LVF/RH presentation and reduced rightlateralization for $\mathrm{RVF} / \mathrm{LH}$ presentation.

Peak latency of the N400 effect in each VF was measured using the difference ERP between 350 and $450 \mathrm{msec}$ at the middle central channel (MiCe), where N400 effects are usually most pronounced, and subjected to an 
omnibus ANOVA on two levels of visual field (RVF vs. LVF). Peak latency was $384 \mathrm{msec}$ for RVF presentation and $390 \mathrm{msec}$ for LVF presentation; these peak latencies did not differ $[F(1,31)=0.65, p=.42] .{ }^{5}$ Because of the importance of latency differences for assessing the possibility of callosal transfer as an explanation for the result pattern, we did an additional analysis comparing, at the 15 central and posterior sites where N400 effects are most prominent, the mean amplitude of the difference ERP across VFs in successive 25 -msec time windows from $200-400 \mathrm{msec}$. There was no significant difference in any of the eight time windows (all $p \mathrm{~s}>.1$ ), suggesting that not only the peak but also the time course of the buildup of the N400 constraint effect was similar for both VFs.

In summary, and as expected, N400 responses were larger for target words in weakly as opposed to strongly constraining sentences. This was true for presentation in both VFs. There was no evidence in any of the comparisons for a difference in the magnitude or latency of the N400 response to the two types of target words as a function of which hemisphere initially received the stimulus.
There was, however, a VF-based skew in the N400 effect distribution over posterior channels.

\section{DISCUSSION}

This experiment was designed to test the claim that the semantic processing of words presented initially to the RH does not benefit from message-level context information. Hypotheses derived from priming measures in lexical decision tasks have predicted a striking difference in the impact of sentential constraint on the processing of sentence-final target words presented in the LVF versus the RVF. Such studies have consistently found that $\mathrm{LVF} / \mathrm{RH}$ word processing is largely insensitive to manipulations of message-level meaning, such as the number (Faust et al., 1993) or order (Faust et al., 1995) of words in the context or the cloze probability of the terminal word (Faust \& Kravetz, 1998). By contrast, these same manipulations do modulate the facilitation observed with initial presentation to the RVF/LH. Furthermore, such data have suggested that when word-level information and message-level information come into conflict, LVF/RH

N400 CONSTRAINT EFFECT:

WEAKLY - STRONGLY CONSTRAINED TARGETS

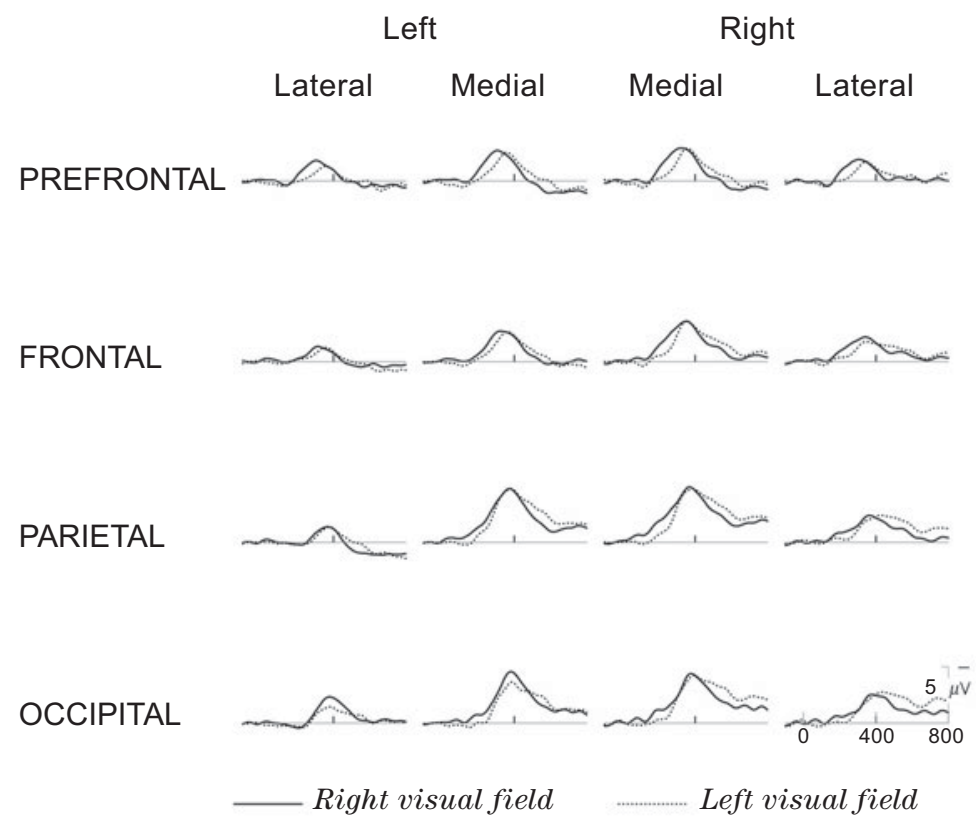

Figure 5. N400 constraint effects. Difference waves are plotted that show the $\mathbf{N 4 0 0}$ constraint effect (responses to targets in weakly constraining contexts minus responses to targets in strongly constraining contexts). The waveforms at the 16 electrode sites (LLPf, LLFr, LLTe, LLOc, LMPf, LDFr, LMCe, LMOc, RMPf, RDFr, RMCe, RMOc, RLPf, RLFr, RLTe, and RLOc) illustrate the distribution of the $\mathrm{N} 400$ effect, which is largest over medial central sites; as is typical for the $\mathbf{N 4 0 0}$, responses also tend to be larger over right than over left scalp sites, although this tendency is increased for presentation to the right hemisphere (LVF). Constraint effects start earlier (in the P2 time window) over frontal sites with RVF presentation, but in the N400 time window (300$500 \mathrm{msec}$ ) are statistically indistinguishable in both amplitude and latency for the two VFs. 


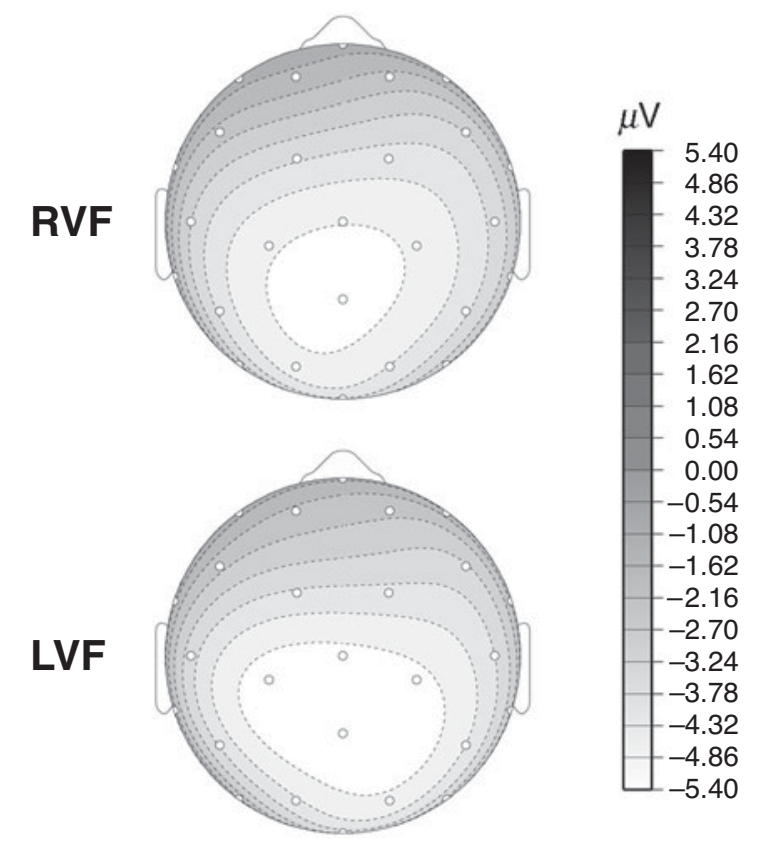

Figure 6. Topography of the $\mathrm{N400}$ effect. Topographic voltage maps (as seen from the top of the head for a 20-msec window around 390 msec) of the $\mathbf{N} 400$ constraint effect are shown for RVF and LVF presentation. Over the posterior part of the scalp, the distribution of the $\mathbf{N 4 0 0}$ effect is skewed toward electrode sites contralateral to the presentation VF (i.e., more right-lateralized after LVF presentation, and vice versa).

measures are primarily driven by the word-level information (e.g., lexical association), whereas RVF/LH measures are primarily driven by the message-level information (Faust et al., 1995). This pattern has been interpreted as showing that the RH can exploit lexical-level context but that the LH is superior in ability to integrate lexical, syntactic, and semantic information to construct a messagelevel representation of meaning (Chiarello et al., 2001; Faust et al., 2003).

In order to test this hypothesis, the present experiment used ERP measures to examine the processing of sentencefinal target words under different degrees of contextual constraint. All target words were plausible completions of the sentences, but the contexts were designed to provide different amounts of message-level constraint on them. In addition, care was taken to ensure that the targets could not be differentially predicted on the basis of simple lexical-level associations. Therefore, divergent patterns of facilitation, here measured using N400 amplitudes, would be expected depending on whether only lexical-level information is processed, as has been hypothesized for LVF/RH stimuli, or a message-level meaning of the sentence is constructed and used, as has been assumed for RVF/LH stimuli. To the extent that only lexical-associative information is available to affect word processing, little if any difference in facilitation should be observed for target words within contexts characterized by strong versus weak message-level constraint. In contrast, to the extent that the message-level meaning of the sentence is constructed and used during a word's processing, more facilitation (greater N400 amplitude reductions) would be expected for words in strongly constraining contexts, as compared with when these same lexical items are embedded in weakly constraining contexts.

As expected, a significant effect of message-level constraint was evident in the N400 responses observed after RVF presentation, with reduced amplitudes to (lexically identical) target words when they were embedded in strongly as opposed to weakly constraining sentences. Strikingly, however, we also observed a significant N400 constraint effect after LVF presentation. To our knowledge, this is the first demonstration that message-level constraint modulates the semantic processing of plausible sentence-final words presented to the LVF/RH. As already described, prior behavioral studies using lexical decision response times have found no effects of constraint or other message-level manipulations for congruent targets in the LVF (although effects on incongruent targets have sometimes been observed). With a different dependent measure, however, we found that messagelevel information can and does impact the semantic processing of stimuli initially presented to the RH. Indeed, not only did we find significant simple effects of constraint for presentation in both VFs, we found no evidence whatsoever that this N400 constraint effect differed in either size or timing as a function of VF. There was thus no indication that message-level information was differentially important for the semantic integration of words presented initially to the right rather than the left hemisphere.

We interpret the significant effect of constraint with LVF presentation as indicating that the $\mathrm{RH}$, like the $\mathrm{LH}$, uses message-level information when processing words. Without additional information, it is probably not reasonable to assume that in neurologically intact adults stimuli presented in one VF will be processed solely by the contralateral hemisphere, since the two hemispheres are connected. Several aspects of our results, however, indicate that hemifield presentation was likely successful in shifting the relative balance of processing toward the contralateral hemisphere, as is generally accepted. First, it is worth noting that VF-based asymmetries in behavioral measures of word processing in young adults are commonplace (see, e.g., reviews by Chiarello, 2003, and Faust, 1998). ${ }^{6}$ Moreover, our prior ERP studies evidenced such asymmetries for the N400 under task conditions identical to those used here (Federmeier \& Kutas, 1999a). Thus, we have every reason to believe a priori that under such conditions visual half-field methods do sample asymmetrically from the two hemispheres when neurally intact college undergraduates read sentences. In fact, in a result that replicates our prior hemifield ERP studies (Federmeier \& Kutas, 1999a, 2002), we found that posterior N1 responses were largest contralateral to presentation VF, demonstrating that relatively early visual processing was lateralized to the contralateral hemisphere. These early sensory potentials were followed by a con- 
tralaterally skewed negative potential (the selection negativity) that extended throughout the $1-\mathrm{sec}$ recording epoch, further demonstrating that at least some aspects of the time-locked neural activity continued to depend on VF during the time frame of all the ERP measures of interest for the present study. Finally, there were differences dependent on VF of presentation even on the delayedrecognition memory task. Thus, at no point in time during the recording epoch was brain processing wholly unaffected by VF of initial presentation.

Of course, the presence of a VF-based asymmetry in and of itself does not rule out the possibility that the languagedominant LH was largely if not solely responsible for all of the semantic context effects observed. Several aspects of the behavior of the N400 response, however, argue against this view. Interhemispheric transfer of information takes time: Callosal relay times have been measured to be on the order of 10-25 $\mathrm{msec}$ (see, e.g., the review by Hoptman \& Davidson, 1994), and ERPs have the temporal resolution to detect such delays. Indeed, we found significant delays of $10 \mathrm{msec}$ on the $\mathrm{N} 1$ component (the ipsilateral portion of which is known to arise from hemispheric transfer [Rugg, Milner, \& Lines, 1985]) and had sufficient power to be able to detect comparable delays on the N400, were they present. We did not, however, find any reliable peak latency differences for the N400 effect, as would have had to occur under the hypothesis that the LH performs the message-level integration regardless of VF of presentation, but only after transfer from the RH following LVF presentation. At the same time, whereas N400 latencies and amplitudes did not differ as a function of VF, the amplitude distribution of the N400 effect across the scalp was skewed toward electrodes over the hemisphere contralateral to presentation VF (greater right lateralization for $\mathrm{LVF} / \mathrm{RH}$ presentation and reduced right lateralization for $\mathrm{RVF} / \mathrm{LH}$ presentation). Rather than finding identical constraint effects that were shifted in time, we found functionally identical effects that were slightly shifted in space (due to nonoverlapping neural generators), in a direction consistent with contralateral processing. Overall, then, multiple aspects of our data strongly support the hypothesis that LVF presentation leads to a greater degree of $\mathrm{RH}$ processing than does RVF presentation, and vice versa.

Our finding that message-level constraint information has a significant impact on LVF-initiated RH word processing contrasts with predictions based on behavioral data collected under similar conditions, although it coheres with the reported absence of any reliable VF-based differences in N400 amplitude reduction for contextually congruent versus wholly incongruent sentence completions (Federmeier \& Kutas, 1999a). The disparity between the behavioral and ERP results clearly merits further study, and a number of differences between the measures, materials, and paradigms that have been used with each could be the basis for the disparity. Arguably, one critical distinction is that our N400s to lateralized target words were recorded as participants simply read for comprehension, without any concomitant speeded overt response. With RT measures for lateralized target words, there is always the potential confound of an overall RVF/ LH advantage for speeded responding in language tasks such as naming and lexical decision. Inferences about the language abilities of the two hemispheres based on lexical decision tasks have been questioned on other grounds as well. As mentioned previously, investigations in split-brain patients have revealed that the lexical decision task, which studies of hemispheric differences in sentence processing have used almost exclusively, underrepresents the semantic abilities of the RH (Baynes \& Eliassen, 1998; Zaidel, 1990). Perhaps, then, the RH is able to use message-level information to process words for comprehension (as reflected in N400 responses obtained during reading as well as in the types of tasks often used in neuropsychological studies) but not to make speeded lexical decision responses. Alternatively, as will be explored in more detail below, the behavioral patterns may reflect asymmetries arising at a different processing level. The functional specificity of the N400 measure for semantic processing allows us to be reasonably confident that the facilitation we observe in both VFs is related to semantic access and integration processes.

In fact, we did find VF-based dissociations for other ERP components and behavioral measures of recognition in the present study. Specifically, we observed VFbased differences between the hemispheres in the effects of constraint on recognition memory for the target words. Target words were more likely to be recognized at the end of the experiment if they had been viewed in weakly constraining sentences, but only for presentation to the $\mathrm{RVF} / \mathrm{LH}$. With presentation to the $\mathrm{LVF} / \mathrm{RH}$, there was no difference in memory for target words as a function of constraint, and memory for strongly constrained targets was not different as a function of VF presentation. There was thus a selective improvement in recognition memory for words presented in the RVF (but not the LVF) if they had originally completed weakly constraining contexts. It seems reasonable to suppose that the decreased predictability of words in less constraining contexts resulted in increased attention and/or elaborative encoding during processing, improving subsequent recognition memory for these items. Indeed, this hypothesis is consistent with our finding, discussed in more detail later, that the earlier P2 component, which has been linked to perception and attentional processes, was affected by constraint only with RVF presentation. Whatever the basis for this effect, however, it occurred only for words initially processed by the LH. Little is known about hemispheric differences in memory function, although a few studies have reported a recognition memory advantage for verbal material presented initially to the RVF (Coney \& MacDonald, 1988; Dee \& Fontenot, 1973). To our knowledge, this study is the first to report a VF-based difference in the effect of contextual variables on recognition memory in brain-intact individuals. More generally, the difference between our ERP and behavioral results makes clear that multiple processes are affected by the VF manipulation and can be affected in different ways. 
In sum, whereas we found constraint-based facilitation of semantic processing in both VFs, constraint-based facilitation of explicit recognition memory performance was found only for RVF presentation.

Although our results demonstrate that message-level information is available and used to facilitate semantic processing for stimuli presented initially to either hemisphere, they need not imply that this message-level information will always impact semantic processing in the same way for the two VFs. Indeed, unexpected sentence endings from the same category as the expected completions (within-category violations) have been shown to elicit different N400 responses in the two VFs (Federmeier \& Kutas, 1999a). When presented to the RVF/LH, these violations elicited smaller N400 responses than did unexpected items from a different semantic category (between-category violations). However, in the LVF/RH, responses to within- and between-category violations were of the same magnitude. The LVF/RH responses mirrored the message-level plausibility of the ending types (which was empirically determined and controlled), but the RVF/LH responses revealed a sensitivity to the featural overlap between an ending that was presented and one that could be expected - but never actually appeared.

On the basis of these findings, we hypothesized that there is a fundamental difference in how the two hemispheres make use of context to create a message-level representation. In particular, we proposed that the $\mathrm{LH}$, but not the RH, uses context information to actively prepare for the processing of likely upcoming stimuli- that is, to preactivate (predict). According to this hypothesis, word processing initiated by either hemisphere is impacted by the message-level representation shaped by the words in the context, among other factors. The RH's message-level representation is made up of information derived directly from the ongoing context in a bottom-up fashion. This representation is compared with the features activated by the word at hand, and the observable facilitation is then primarily a function of that fit (e.g., how well baseball coheres with context features such as pass and touchdown). In the LH, in contrast, processing reflects a bidirectional interplay between bottom-up and top-down processing of the ongoing sentence context, the consequence of which is the preactivation of semantic, and possibly even perceptual, features of likely upcoming words, and the observable facilitation is a function of the overlap between the target actually presented and that contextually derived prediction (e.g., how similar baseball is to the predicted football-on all salient features, not just those highlighted by the context).

We cannot assess this hypothesis with the N400 data from the present study, since increased contextual constraint could be expected to aid the semantic processing of target words under both "integration" (RH) and "expectancy" (LH) strategies. Certainly, a more constraining context would seem to allow for better prediction, by placing more constraints on the set of likely upcoming words and/or concepts. Nonetheless, constraint effects could also emerge without predictive processing, since more constraining contexts are likely to provide more and/or better information with which to integrate incoming stimuli. In this study, we found no evidence that the use of constraining contextual information differentially affected semantic aspects of word processing as a function of VF of presentation. However, other aspects of the present data do relate to our hypothesis that RVF presentation triggers a predictive strategy that LVF presentation does not. Specifically, we observed constraintbased VF effects on the P2 component of the ERP. ${ }^{7}$

The P2 is a fronto-central positivity peaking around $250 \mathrm{msec}$ that often coincides with the onset of an N400 effect. Both the precise functional significance of the P2 component and its neural source remain imperfectly understood. Amplitude differences in the P2, however, typically have been linked to the detection and analysis of visual features in attention paradigms, with increased amplitudes for stimuli containing target features (Hillyard \& Münte, 1984; Luck \& Hillyard, 1994); P2 thus seems to reflect some stage of higher order visual processing. In the present experiment, we observed an interaction between constraint and VF. P2s to words in weakly constraining contexts did not differ with VF, but those to words in strongly constraining contexts were larger (more positive) for $\mathrm{RVF} / \mathrm{LH}$ presentation. This pattern is reminiscent of that observed for congruency effects with sentence-final pictures: larger P2s for congruent pictures, but only with initial presentation to the LH (Federmeier \& Kutas, 2002). This pattern is also similar to behavioral effect patterns, in which contextual information facilitates response speed for RVF but not LVF words. If these patterns are indeed linked, it would indicate that the behavioral asymmetries arise primarily from perceptual, rather than semantic, processing differences, in contrast to what is typically assumed. Our P2 findings thus suggest that the message-level information previously extracted from a sentence context can provide top-down information, which in turn allows for more efficient visual extraction from expected, and especially from highly constrained, target stimuli. This facilitative effect of predictive language information on higher level visual processing seems, however, to occur only for stimuli initially processed by the $\mathrm{LH}$; recall that these words were also generally less well remembered.

In summary, then, we found that the semantic processing of a visual word stimulus is influenced by the messagelevel constraint of a sentence context for initial presentation to either the LH or RH. This, in turn, suggests that the asymmetries found with behavioral measures for the impact of sentence-level information on the processing of LVF and RVF words do not arise because RH semantic processing has no or less access to message-level meaning information, as has been commonly thought. At the same time, however, we found that higher order perceptual visual processing was more affected by sentential constraint with initial presentation to the LH, in accord with the hypothesis that the LH (but not the RH) uses context information to predict the semantic and perceptual features of likely upcoming words. VF-based 
differences were also revealed in participants' recognition memory for the lateralized target words, which was affected by constraint only for RVF/LH presentation. Hemispheric asymmetries thus seem to arise at multiple processing levels and to manifest differently across tasks, which may help to explain disparities in the conclusions that have been drawn about RH language capacities from neuropsychological, behavioral, and electrophysiological measures. Thus far, ERP data are consistent with the hypothesis that both hemispheres make use of higher level meaning information to guide semantic processing, although they likely use such information differently.

\section{REFERENCES}

BANICH, M. T. (2002). The divided visual field technique in laterality and interhemispheric integration. In K. Hugdahl (Ed.), Experimental methods in neuropsychology. Boston: Kluwer.

BANICH, M. T. (2003). Interaction between the hemispheres and its implications for the processing capacity of the brain. In R. Davidson and K. Hugdahl (Eds.), Brain asymmetry (2nd ed., pp. 261-302). Cambridge, MA: MIT Press.

Baynes, K., \& Eliassen, J. C. (1998). The visual lexicon: Its access and organization in commissurotomy patients. In M. Beeman \& C. Chiarello (Eds.), Right hemisphere language comprehension: Perspectives from cognitive neuroscience (pp. 79-104). Mahwah, NJ: Erlbaum.

BeEman, M. (1993). Semantic processing in the right hemisphere may contribute to drawing inferences from discourse. Brain \& Language, 44, 80-120.

Beeman, M. (1998). Coarse semantic coding and discourse comprehension. In M. Beeman \& C. Chiarello (Eds.), Right hemisphere language comprehension: Perspectives from cognitive neuroscience (pp. 255-284). Mahwah, NJ: Erlbaum.

Beeman, M., Friedman, R. B., Grafman, J., \& Perez, E. (1994). Summation priming and coarse semantic coding in the right hemisphere. Journal of Cognitive Neuroscience, 6, 26-45.

Bentin, S., McCarthy, G., \& Wood, C. C. (1985). Event-related potentials associated with semantic priming. Electroencephalography \& Clinical Neurophysiology, 60, 343-355.

Berardi, N., \& Fiorentini, A. (1997). Interhemispheric transfer of spatial and temporal frequency information. In S. Christman (Ed.), Cerebral asymmetries in sensory and perceptual processing (pp. 5579). New York: Elsevier.

Bloom, P. A., \& Fischler, I. (1980). Completion norms for 329 sentence contexts. Memory \& Cognition, 8, 631-642.

Bottini, G., Corcoran, R., Sterzi, R., Paulesu, E. S., Schenone, P., Scarpa, P., Frackowiak, R. S. J., \& Frith, C. D. (1994). The role of the right hemisphere in the interpretation of figurative aspects of language: A positron emission tomography activation study. Brain, 117, 1241-1253.

Brownell, H. H., Michel, D., Powelson, J., \& Gardner, H. (1983). Surprise but not coherence: Sensitivity to verbal humor in righthemisphere patients. Brain \& Language, 18, 20-27.

Brownell, H. H., Potter, H. H., Bihrle, A. M., \& Gardner, H. (1986). Inference deficits in right brain-damaged patients. Brain \& Language, 27, 310-321.

Bub, D. N., \& ARguin, M. (1995). Visual word activation in pure alexia. Brain \& Language, 49, 77-103.

Burgess, C., \& Simpson, G. B. (1988). Cerebral hemispheric mechanisms in the retrieval of ambiguous word meanings. Brain \& Language, 33, 86-103.

Chiarello, C. (2003). Parallel systems for processing language: Hemispheric complementarity in the normal brain. In M. T. Banich \& M. Mack (Eds.), Mind, brain, and language: Multidisciplinary perspectives (pp. 229-247). Mahwah, NJ: Erlbaum.

Chiarello, C., Burgess, C., Richards, L., \& Pollock, A. (1990). Semantic and associative priming in the cerebral hemispheres: Some words do, some words don't . . . sometimes, some places. Brain \& Language, 38, 75-104.

Chiarello, C., Liu, S., \& Faust, M. (2001). Bihemispheric sensitivity to sentence anomaly. Neuropsychologia, 39, 1451-1463.

Coney, J., \& MacDonald, S. (1988). The effect of retention interval upon hemispheric processes in recognition memory. Neuropsychologia, 26, 287-295.

Dee, H. L., \& Fontenot, D. J. (1973). Cerebral dominance and lateral differences in perception and memory. Neuropsychologia, 11, 11671173.

Delis, D. C., Wapner, W., Gardner, H., \& Moses, J. A. (1983). The contribution of the right hemisphere to the organization of paragraphs. Cortex, 19, 43-50.

FAUST, M. (1998). Obtaining evidence of language comprehension from sentence priming. In M. Beeman \& C. Chiarello (Eds.), Right hemisphere language comprehension: Perspectives from cognitive neuroscience (pp. 161-185). Mahwah, NJ: Erlbaum.

Faust, M., BabKoff, H., \& Kravetz, S. (1995). Linguistic processes in the two cerebral hemispheres: Implications for modularity vs. interactionism. Journal of Clinical \& Experimental Neuropsychology, 17, 171-192.

Faust, M., Bar-Lev, A., \& Chiarello, C. (2003). Sentence priming effects in the two cerebral hemispheres: Influences of lexical relatedness, word order, and sentence anomaly. Neuropsychologia, 41, 480-492.

Faust, M., \& Kravetz, S. (1998). Levels of sentence constraint and lexical decision in the two hemispheres. Brain \& Language, 62, 149-162.

Faust, M., Kravetz, S., \& BabKoff, H. (1993). Hemisphericity and top-down processing of language. Brain \& Language, 44, 1-18.

FEDERMEIER, K. D., \& KutAs, M. (1999a). Right words and left words: Electrophysiological evidence for hemispheric differences in meaning processing. Cognitive Brain Research, 8, 373-392.

Federmeier, K. D., \& Kutas, M. (1999b). A rose by any other name: Long-term memory structure and sentence processing. Journal of Memory \& Language, 41, 469-495.

Federmeier, K. D., \& Kutas, M. (2002). Picture the difference: Electrophysiological investigations of picture processing in the cerebral hemispheres. Neuropsychologia, 40, 730-747.

Francis, W. N., \& KučERA, H. (1982). Frequency analysis of English usage. Boston: Houghton Mifflin.

Gardner, H., Brownell, H. H., Wapner, W., \& Michelow, D. (1983). Missing the point? The role of the right hemisphere in the processing of complex linguistic materials. In E. Perecman (Ed.), Cognitive processing in the right hemisphere (pp. 169-191). New York: Academic Press.

Gough, P. B., Alford, J. A., JR., \& Holley-Wilcox, P. (1981). Words and contexts. In O. J. L. Tzeng \& H. Singer (Eds.), Perception of print: Reading research in experimental psychology (pp. 85-102). Hillsdale, NJ: Erlbaum.

Griffin, Z. M., \& Bock, K. (1998). Constraint, word frequency, and the relationship between lexical processing levels in spoken word production. Journal of Memory \& Language, 38, 313-338.

Hillyard, S. A., \& MüNTE, T. F. (1984). Selective attention to color and location: An analysis with event-related brain potentials. Perception \& Psychophysics, 36, 185-198.

Hoptman, M. J., \& Davidson, R. J. (1994). How and why do the two cerebral hemispheres interact? Psychological Bulletin, 116, 195-219.

Joanette, Y., Goulet, P., \& Hannequin, D. (1990). Right hemisphere and verbal communication. New York: Springer.

Kiss, G. R., Armstrong, C., Milroy, R., \& Piper, J. (1973). An associative thesaurus of English and its computer analysis. In A. J. Aitkin, R. W. Bailey, \& N. Hamilton-Smith (Eds.), The computer and literary studies (pp. 153-165). Edinburgh: Edinburgh University Press.

KuTAS, M., \& FEDERMEIER, K. D. (2001). Electrophysiology reveals semantic memory use in language comprehension. Trends in Cognitive Sciences, 4, 463-470.

KutAs, M., \& Hillyard, S. A. (1980a). Event-related brain potentials to semantically inappropriate and surprisingly large words. Biological Psychology, 11, 99-116.

Kutas, M., \& Hillyard, S. A. (1980b). Reading senseless sentences: Brain potentials reflect semantic incongruity. Science, 207, 203-205. Kutas, M., \& Hillyard, S. A. (1983). Event-related brain potentials to 
grammatical errors and semantic anomalies. Memory \& Cognition, 11, 539-550.

Kutas, M., \& Hillyard, S. A. (1984). Brain potentials during reading reflect word expectancy and semantic association. Nature, 307, 161163.

Kutas, M., Lindamood, T. E., \& Hillyard, S. A. (1984). Word expectancy and event-related brain potentials during sentence processing. In S. Kornblum \& J. Requin (Eds.), Preparatory states and processes (pp. 217-237). Hillsdale, NJ: Erlbaum.

LUCK, S. J., \& HiLlYARD, S. A. (1994). Electrophysiological correlates of feature analysis during visual search. Psychophysiology, 31, 291-308.

MARTIN, R. C. (2003). Language processing: Functional organization and neuroanatomical basis. Annual Review of Psychology, 54, 55-89.

NAGY, M. E., \& RUGG, M. D. (1989). Modulation of event-related potentials by word repetition: The effects of inter-item lag. Psychophysiology, 26, 431-436.

Neville, H. J., Kutas, M., \& Schmidt, A. (1982). Event-related potential studies of cerebral specialization during reading: I. Studies of normal adults. Brain \& Language, 16, 300-315.

Ni, W., Constable, R. T., Mencl, W. E., Pugh, K. R., Fulbright, R. K., Shaywitz, S. E., Shaywitz, B. A., Gore, J. C., \& Shankweiler, D. (2000). An event-related neuroimaging study distinguishing form and content in sentence processing. Journal of Cognitive Neuroscience, 12, 120-133.

OldFIELD, R. C. (1971). The assessment and analysis of handedness: The Edinburgh inventory. Neuropsychologia, 9, 97-113.

RatclifF, R., \& McKoon, G. (1988). A retrieval theory of priming in memory. Psychological Review, 95, 385-408.

RAYNER, K., \& WeLL, A. D. (1996). Effects of contextual constraint on eye movements in reading: A further examination. Psychonomic Bulletin \& Review, 3, 504-509.

Robertson, D. A., Gernsbacher, M. A., Guidotti, S. J., Robertson, R. R. W., Irwin, W., Mock, B. J., \& Campana, M. E. (2000). Functional neuroanatomy of the cognitive process of mapping during discourse comprehension. Psychological Science, 11, 255-260.

RugG, M. D., Milner, A. D., \& Lines, C. R. (1985). Visual evoked potentials to lateralised stimuli in two cases of callosal agenesis. Journal of Neurology, Neurosurgery, \& Psychiatry, 48, 367-373.

SANOCKI, T., \& ODEN, G. C. (1984). Contextual validity and the effects of low-constraint sentence contexts on lexical decisions. Quarterly Journal of Experimental Psychology, 36A, 145-156.

Schwanenflugel, P. J., \& SHoben, E. J. (1985). The influence of sentence constraint on the scope of facilitation for upcoming words. Journal of Memory \& Language, 24, 232-252.

St. George, M., Kutas, M., Martinez, A., \& Sereno, M. I. (1999). Semantic integration in reading: Engagement of the right hemisphere during discourse processing. Brain, 122, 1317-1325.

TAYLOR, W. L. (1953). "Cloze procedure": A new tool for measuring readability. Journalism Quarterly, 30, 415-433.

Titone, D. (1998). Hemispheric differences in context sensitivity during lexical ambiguity resolution. Brain \& Language, 65, 361-394.

Van Petten, C. (1993). A comparison of lexical and sentence-level context effects in event-related potentials. Language \& Cognitive Processes, 8, 485-531.

Van Petten, C., \& Kutas, M. (1990). Interactions between sentence context and word frequency in event-related brain potentials. Memory \& Cognition, 18, 380-393.

WAPNER, W., HAMBY, S., \& GARDNER, H. (1981). The role of the right hemisphere in the apprehension of complex linguistic materials. Brain \& Language, 14, 15-33.

Weylman, S. T., Brownell, H. H., Roman, M., \& Gardner, H. (1989). Appreciation of indirect requests by left- and right-brain-damaged patients: The effects of verbal context and conventionality of wording. Brain \& Language, 36, 580-591.

ZAIDEL, E. (1990). Language functions in the two hemispheres following complete cerebral commissurotomy and hemispherectomy. In F. Boller \& J. Grafman (Series Eds.) \& R. D. Nebes \& S. Corkin (Vol. Eds.), Handbook of neuropsychology (Vol. 4, pp. 115-150). Amsterdam: Elsevier.

\section{NOTES}

1. The cloze probability for a given word in a given sentence context is defined as the proportion of individuals choosing to complete that particular context with that particular word (Taylor, 1953).

2. Equal numbers of each constraint condition were shown to each hemisphere in an experimental session. Order of presentation was randomized with the stipulation that no more than three stimuli in a row be presented to the same hemifield.

3. This is the same effect previously described for the late, lateralized negativity, and it may reflect overlap of the two responses.

4. In order to examine scalp distribution, we used the standard procedure of dividing scalp electrodes into regions (left/right, medial/lateral, anterior/posterior). Not all electrode sites can be used (e.g., the four midline channels cannot be classified with respect to hemisphere); here, we used 16. Left lateral sites were (from front to back) LLPf, LLFr, LLTe, and LLOc. Left medial sites were LMPf, LMFr, LMCe, and LMOc. Right medial sites were RMPf, RMFr, RMCe, and RMOc. Right lateral sites were RLPf, RLFr, RLTe, and RLOc.

5. There were also no peak latency differences at more posterior $[\mathrm{MiPa}: F(1,31)=0.61, p=.44]$ or more frontal [MiPf: $F(1,31)=0.84$, $p=.37$ ] channels. The standard deviation of $\mathrm{N} 400$ peak effect latency is typically around $30 \mathrm{msec}$ for measurements made at a single channel. For the test employed here (within-subjects ANOVA with two levels of one factor), we had statistical power of 0.6 to see differences of $12 \mathrm{msec}$ (the value typically measured for sensory components; Hoptman \& Davidson, 1994) and power of 1.0 to see differences greater than $25 \mathrm{msec}$ (which is in the upper range of measured differences).

6 . The task used for this study, reading for comprehension, is arguably even more likely than behavioral measures to reflect laterally biased processing, since lexical decision judgments seem to be more difficult for the isolated RH than semantic comprehension (Baynes \& Eliassen, 1998; Zaidel, 1990), and studies in other domains have shown that levels of interhemispheric cooperation tend to increase with task difficulty (Banich, 2003).

7. Because of the potential for temporal overlap between P2 and N400 effects, it is important to ascertain that the earlier effect of constraint for the LVF stimuli reflects something other than an earlier onset of the N400 constraint effect. Since P2 effects have a more frontal scalp distribution than do N400 effects, a distributional analysis can help make this differentiation. We compared the RVF constraint effect in the two time windows (200-300 msec and 300-500 msec) using an omnibus 2 (time window) $\times 2$ (anteriority: 11 frontal channels, as already described for the $\mathrm{P} 2$ analysis, and 11 posterior channels, including those used in the $\mathrm{N} 1$ analysis plus MiOc) $\times 11$ (electrode) ANOVA. In addition to a main effect of time window $[F(1,31)=6.01, p=.02]$, with a larger effect between 300 and $500 \mathrm{msec}$, we observed a significant crossover interaction between time window and anteriority $[F(1,31)=$ $23.57, p<.001]$, with larger effects over frontal than over posterior sites between 200 and $300 \mathrm{msec}$ but larger effects over posterior than over frontal sites between 300 and $500 \mathrm{msec}$. A split within the $300-$ to $500-\mathrm{msec}$ window itself did not yield this distributional interaction $[F(1,31)=$ $0.64, p=.43]$. Thus, the effect between 200 and $300 \mathrm{msec}$ does not seem to be simply an earlier onset of the N400 effect and, in fact, has a scalp distribution consistent with that typically observed for P2. 


\section{APPENDIX}

Examples of Stimuli Used in the Experiment

One hundred sixty target words were each paired with a strongly (S) and a weakly (W) constraining sentence context. Below are 30 representative examples of these stimuli, with target words in italics. Note that individual participants saw each target word only once.

S: $\quad$ She was suddenly called back to New York and had to take a cab to the airport.

$\mathrm{W}$ : She was glad she had brought a book since there was nothing to read at the airport.

S: The magician took out his hat and made a rabbit appear.

W: At the end of the grand feast, the queen waited patiently for the man to appear.

S: $\quad$ My grandmother keeps her wedding gown and old photos in the attic.

W: Upon mother's strict orders, the cat's smelly litter box was kept in the attic.

S: $\quad$ No one at the reunion recognized Dan because he had grown a beard.

W: At the children's park next to the beach she saw a man with a beard.

S: He was cold most of the night and finally got up to get another blanket.

W: They decided to declaw the feisty cat so he wouldn't tear up the blanket.

S: Tracy and her husband weren't Catholic, but she was glad when the priest warmly welcomed their three children.

$\mathrm{W}$ : She bought several colorful and scenic postcards to send to her children.

$\mathrm{S}$ : Within minutes of arriving at the office, the detective spilled his thermos full of coffee.

W: After getting out of the hot shower he sat down to have coffee.

S: The tenants decided to take their landlord to court.

W: He wanted to sit in the front of the court.

S: He didn't worry about burglars because he kept two fierce dogs.

$\mathrm{W}$ : They both have jobs, but they get some extra income by raising dogs.

S: $\quad$ They stood on the bridge tossing stale bread to the ducks.

W: He grew corn in one of his expansive fields to feed his ducks.

S: The janitor saw the vacuum cleaner bag explode and was afraid the room would soon be covered in dust.

W: He finished washing the second load of laundry and started to dust.

S: The man who was carried into the emergency room had been stabbed in a fight.

W: Sam practiced at his friend's house every day in anticipation of the fight.

S: $\quad$ The white gull dove down and caught a fish.

W: They had to drive for another hour before finding a place to fish.

S: He tried to put the pieces of the broken plate back together with glue.

$\mathrm{W}$ : She walked across the large room to Mike's messy desk and returned his glue.

S: Most television shows last about an hour.

W: Liz and Marie sat at the large conference table, and they discussed the topic for the next hour.

S: $\quad$ Marie would have been here but she never received the invitation.

$\mathrm{W}$ : He was so busy and overwhelmed that he forgot to respond to the invitation.

S: When George couldn't get into his car, he got down on one knee and reached under the bumper for the extra key.

W: He smiled nervously at the young detective as he searched through his pockets for a key.

S: $\quad$ The cold drink was served with a slice of lemon.

W: The only food left in the barren refrigerator was a moldy lemon.

$\mathrm{S}$ : $\quad$ At the grocery store I always wind up in the slowest moving line.

$\mathrm{W}$ : The young couple's three little girls enjoyed playing together in the line.

S: $\quad$ To help everyone find her house, Lisa drew a map.

W: She helped him out with his project by putting the finishing touches on the map.

S: $\quad$ Before she arrived he opened a bottle of wine and put on some soft music.

W: She took the baby out when he started to cry and had to miss the rest of the music.

S: She picked up a wallet on the street and was honest enough to try to locate the owner.

$\mathrm{W}$ : Once he finished organizing all the binders he returned the hole punch to the owner.

S: $\quad$ The game was called because of rain.

W: She wore heavy wool socks and her sturdiest boots to go walking in the rain. 


\section{APPENDIX (Continued)}

S: $\quad$ She wished she had brought something to read.

W: She asked her distracted son what he wanted to read.

S: $\quad$ Every morning Susan braids her little girl's hair then helps her dress for school.

W: He called early in the morning to arrange his monthly appointment at the school.

S: He never let Fluffy sleep on his couch because she shed.

$\mathrm{W}$ : The cat was crouched in the tall grass down by the shed.

S: Peter painted the walls of the bird exhibit light blue so that it would resemble the sky.

$\mathrm{W}$ : Before going out on the open seas they always checked the sky.

S: He can't be held responsible for the killing because he was crazy at the time.

$\mathrm{W}$ : I started to put new plants in my garden but ran out of time.

S: $\quad$ She pulled her head out from under the faucet and reached for a towel.

$\mathrm{W}$ : After politely standing in line at the hotel desk, the boy asked for another towel.

S: The construction worker had developed very powerful arms from unloading bags of cement from the truck.

W: Nobody knew why there were a lot of old boxes stored in the truck.

(Manuscript received August 20, 2003;

revision accepted for publication September 2, 2004.) 